\title{
Variability of ultraluminous X-ray sources in the Cartwheel Ring
}

\author{
Anna Wolter, ${ }^{1}$ Ginevra Trinchieri ${ }^{1}$ and Monica Colpi ${ }^{2}$ \\ ${ }^{1}$ INAF, Osservatorio Astronomico di Brera, via Brera 28, 20121 Milano, Italy \\ ${ }^{2}$ Dipartimento di Fisica G. Occhialini, Università degli Studi di Milano Bicocca, \\ Piazza della Scienza 3, 20126 Milano, Italy
}

email: anna.wolter@brera.inaf.it, ginevra.trinchieri@brera.inaf.it, Monica.Colpi@mib.infn.it

\begin{abstract}
The Cartwheel is one of the most outstanding examples of a dynamically perturbed galaxy where star formation is occurring inside the ring-like structure. In previous studies with Chandra, we detected 16 Ultra Luminous X-ray sources lying along the southern portion of the ring. Their Luminosity Function is consistent with them being in the high luminosity tail of the High Mass X-ray Binaries distribution, but with one exception: source N.10. This source, detected with Chandra at $L_{X}=1 \times 10^{41} \mathrm{erg} \mathrm{s}^{-1}$, is among the brightest non-nuclear sources ever seen in external galaxies. Recently, we have observed the Cartwheel with XMM-Newton in two epochs, six months apart. After having been at its brightest for at least 4 years, the source has dimmed by at least a factor of two between the two observations. This fact implies that the source is compact in nature. Given its extreme isotropic luminosity, there is the possibility that the source hosts an accreting intermediate-mass black hole. Other sources in the ring vary in flux between the different datasets. We discuss our findings in the context of ULX models.
\end{abstract}

Keywords. Accretion, accretion disks - black hole physics - galaxies: individual (Cartwheel) galaxies: stellar content - X-rays: binaries - X-rays: galaxies

\section{Introduction}

Very luminous off-nuclear X-ray sources in nearby galaxies are known since the Einstein satellite times. They have been named Ultra-Luminous X-ray sources (ULXs) because their isotropic X-ray luminosity is significantly higher than the Eddington limit for a solar mass black hole $\left(L_{X} \sim 1.4 \times 10^{38} \mathrm{erg} \mathrm{s}^{-1}\right)$. The name itself is only a phenomenological description of their $L_{X}$, since their nature is not clear yet. They do not appear to have an equivalent among Galactic sources, and this may be related to the low Star-Formation rate of the Milky Way since ULXs are mostly found associated with Star-Forming regions. Explanations of their nature involve beamed emission from an accreting stellar mass compact object, or super-Eddington emission, or isotropic accretion onto an intermediate-mass black hole.

An extraordinary example of ULX is the source N.10 detected in the narrow, gasrich star-forming ring of the Cartwheel galaxy with isotropic luminosity of $L_{0.5-10 \mathrm{keV}} \sim$ $1.3 \times 10^{41} \mathrm{erg} \mathrm{s}^{-1}$ (Wolter et al. 1999; Wolter \& Trinchieri 2004 - hereafter WT04; Gao et al. 2003). This is the brightest of a number of very bright individual sources that also appear to reside in the ring, with isotropic luminosities in excess of $L_{0.5-10 \mathrm{keV}}=3 \times 10^{39}$ $\operatorname{erg~s}^{-1}$ (WT04).

Source N.10 is one of the brightest ULXs known, with observed $L_{X}$ comparable with the peak $L_{X}$ of the best studied example of the class, source X-1 in M82 (Ptak \& Griffiths 1999; Matsumoto et al. 2001; Kaaret et al. 2001; Strohmayer \& Mushotzky 2003; Dewangan et al. 2006). The spectral and temporal variability of X-1 (typically 


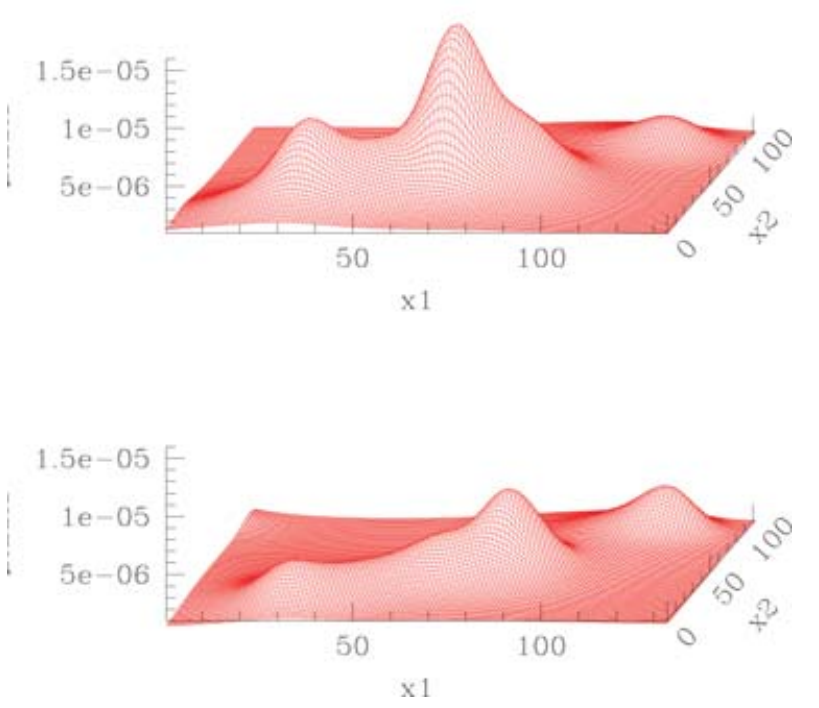

Figure 1. A 3-d representation of the two XMM-Newton pn observations [101] (top) and [201] (bottom) with a six months time separation. The smoothed images have been normalized to the respective exposure time. It is evident that the peak located at the position of source N.10 is very less prominent in the second epoch.

bursts of about a month duration) have led to the estimate of $200 \mathrm{M}_{\odot}$ for the accreting object responsible for the X-ray emission (Dewangan et al. 2006).

The lack of detailed information on variability for source N.10 in the Cartwheel has instead prevented up to now the exclusion of an extended nature. We present here new $X M M-N e w t o n$ observations which have now confirmed its compact nature.

\section{XMM-Newton observations}

We have observed the source with XMM-Newton in two epochs (December 2004 and May 2005). A detailed description of the results obtained for source N.10 is presented in Wolter, Trinchieri \& Colpi (2006). A 3-d representation of the two smoothed datasets is plotted in Figure 1.

The detection of variability is important because it is our strongest proof that we are detecting a single source, namely an accreting binary, and not a collection of less luminous unresolved X-ray sources. It is also relevant because it can provide essential information about the details of the accretion process and, most important, about the masses involved in the process (donor and accretor), giving the possibility to infer the presence of an intermediate-mass black hole.

The spectrum in the two different XMM-Newton observation (the first, [101] of 24 ksec, and the second [201], of 42 ksec exposure) is shown in Figure 2. The XMM-Newton $\mathrm{PSF}$ is such that the extraction radius (even if we used a smaller than customary value of $10^{\prime \prime}$ ) contains part of the ring emission, which is described by the sum of a diffuse gas component (thermal spectrum) and the contribution from the unresolved point-like sources (X-ray binaries; power law spectrum. See WT04 for details). Even if the statistics is low, therefore, a multicomponent spectrum has been used to fit the emission: we have 

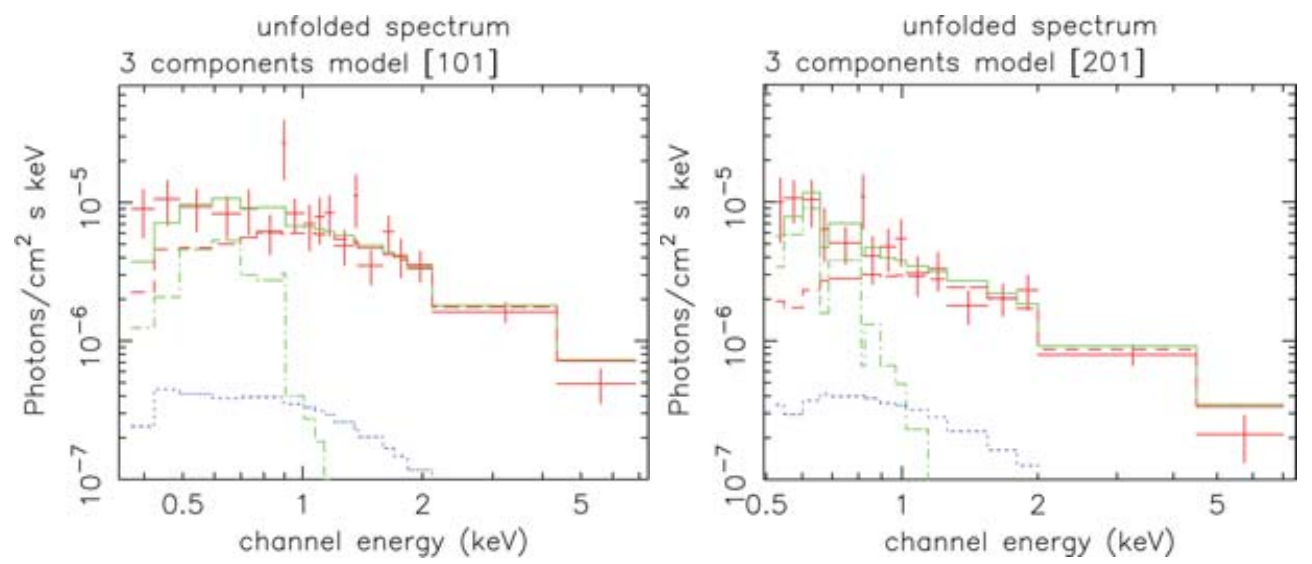

Figure 2. Unfolded spectrum, from pn data only for clarity, showing the three components (green dot-dashed line = gas; blue short dashed line $=$ unresolved binaries; red long dashed line $=$ ULX) at the best fit values. The difference between the left [101] and the right [201] datasets is only the normalization of the 'ULX' component [besides the binning scheme due to the different statistics at the two epochs].

added a power law describing the ULX power law emission (as seen in Chandra) to the two ring components. The spectra of the two observations 6 months apart clearly show that the thermal component (gas in the ring) is constant in time and that the factor of 2 variability of the ULX is quite evident.
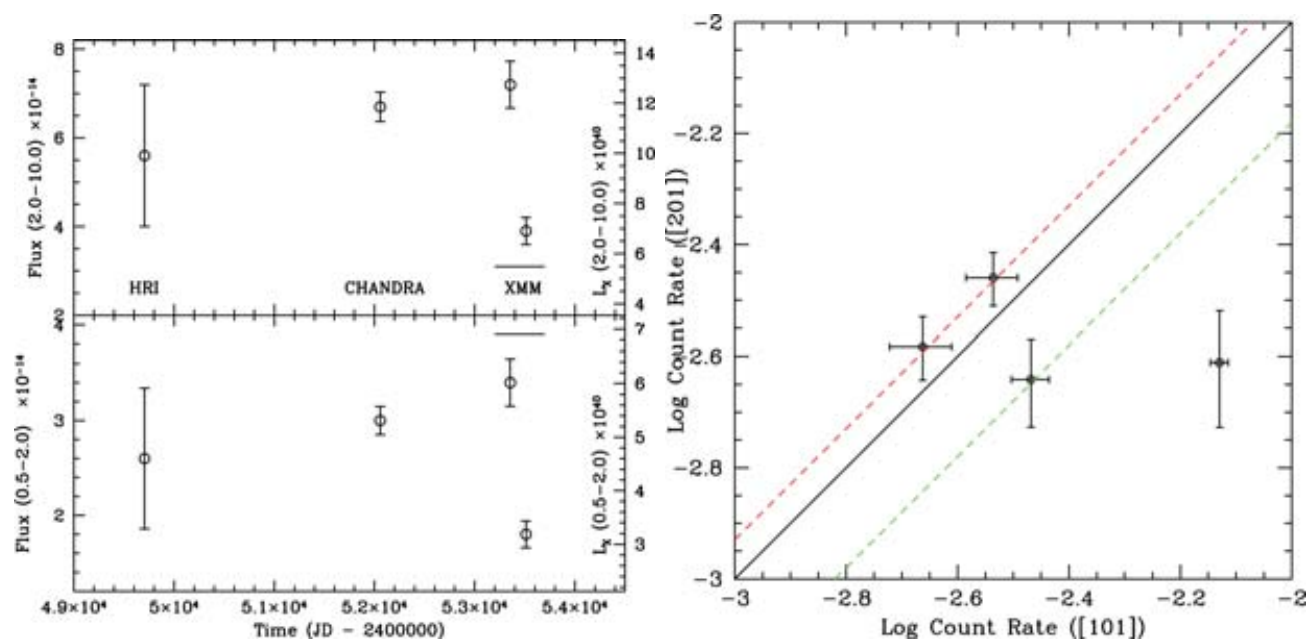

Figure 3. Left: long term light curve in the soft (lower panel) and hard (upper panel) energy band, over an interval of about 10 years. The two XMM-Newton points that define the variation are not subject to cross-calibration uncertainties. All fluxes are computed with the same spectral shape, i.e. power law with $\Gamma=1.6$ and $\mathrm{N}_{\mathrm{H}}=3.6 \times 10^{21} \mathrm{~cm}^{-2}$; see fit to Chandra data in WT04. Right axis reports luminosities computed assuming the Cartwheel distance. From Wolter et al. 2006. Right: flux comparison of the brightest sources in the first and second epoch.

The long term behavior of N.10 shows a nearly constant luminosity for about 10 years (see Figure 3 - left), and then a rapid dimming of at least a factor of 2 in 6 months. This is quite different from the variability pattern observed in the best studied bright ULX, namely $\mathrm{X}-1$ in the starburst galaxy M82 (Dewangan et al. 2006). 
We compared count rates for the brightest source neighboring N.10. The comparison is shown in Figure 3 (Right). We have determined that the source next to it, which corresponds to Chandra sources N.13 \& N.14, has not varied between the two observations (the count rate in the second observation is at most $15 \%$ higher than in the first one). If we assume that this is constant, then the source to the NW, corresponding to N.16 \& N.17, is also constant (the same $15 \%$ increase in the count rate), but the SE source (N.7 $\&$ N.9) has faded to about half its strength in the second observation.

\section{Conclusions}

The whole ring of the Cartwheel consists of bubbles and condensations (Struck et al. 1996) and the neighborhood of N.10 is no exception; the association with an environment of massive and young stars is almost certain. The most appealing interpretation for the emission of source N.10 is that it is powered by an intermediate-mass black hole. We cannot rule out the presence of Super-Eddington accretion. Other sources have been observed to vary in the same time frame of N.10. Further studies on variability will help us in distinguishing between different accretion modes. It is evident that the Cartwheel ring is an excited (and exciting!) environment, in which star formation is violently at work. The low metallicity measured for the optical gas might be a key ingredient for the formation of sources capable of emitting very high X-ray luminosity, whatever the mechanism is.

\section{Acknowledgements}

We acknowledge partial financial support from the Italian Space Agency (ASI) under contract ASI-INAF I/023/05/0. This research has made use of SAOImage DS9, developed by the Smithsonian Astrophysical Observatory. This work is based on observations obtained with XMM-Newton, an ESA science mission with instruments and contributions directly funded by ESA Member States and the USA (NASA).

\section{References}

Dewangan, G. C., Titarchuk, L. \& Griffiths, R. E. 2006, ApJ, 637, L21

Gao, Y., Wang, Q. D., Appleton, P. N. \& Lucas, R. A. 2003, ApJ, 596, 171

Kaaret, P. et al. 2001, MNRAS, 321, L29

Matsumoto, H., Tsuru, T. G., Koyama, K., Awaki, H., Canizares, C. R., Kawai, N. et al. 2001, ApJ, 547, 25

Ptak, A. \& Griffiths, R. 1999, ApJ, 517, L1

Strohmayer, T. E. \& Mushotzky, R. F. 2003, ApJ, 586, L61

Struck, C., Appleton, P. N., Borne, K. D. \& Lucas, R. A. 1996, AJ, 112, 1868

Wolter, A., Trinchieri, G. \& Iovino, A. 1999, A\&A, 342, 41

Wolter, A. \& Trinchieri, G. 2004, A\&A, 426, 787, WT04

Wolter, A., Trinchieri, G. \& Colpi, M. 2006, MNRAS, 373, 1627 